\title{
Estimation of the Stage of Search Process for the Simplex Invariant Method
}

\author{
Artem A. Golub* \\ Siberian Federal University \\ Krasnoyarsk, Russian Federation
}

Received 04.06.2019, received in revised form 10.10.2019, accepted 21.02.2020

Abstract. The article describes the synthesis of rules used for estimating the stage of searching for the simplex invariant method. Computational experiments were carried out on a non-stationary static model. Characteristics of the optimum search and tracking process were obtained. The characteristics were analyzed. Based on the obtained patterns rules were developed that allow evaluating the stage of the search process: movement towards the optimum or its tracking. In conclusion, a summary of the research is given.

Keywords: simplex search, simplex invariant method, direct optimization methods, target function drift, optimization, adaptation, parametric adaptation.

Citation: Golub A.A. Estimation of the stage of search process for the simplex invariant method, J. Sib. Fed. Univ. Eng. \& Technol., 2020, 13(2), 175-186. DOI: 10.17516/1999-494X-0214

\section{Оценка этапа поиска}

\section{симплексного инвариантного метода}

\author{
А.А. Голуб \\ Сибирский федеральный университет \\ Российская Федераџия, Красноярск
}

Аннотация. В статье рассмотрено построение блока правил для оценки этапа поиска симплексного инвариантного метода. Проведены вычислительные эксперименты на нестационарной статической модели, получены характеристики процесса поиска и отслеживания оптимума. Характеристики подвергнуты анализу. На основе полученных закономерностей разработаны правила, позволяющие оценить состояние поиска: движение к цели или ее отслеживание. В заключении дается обобщение проведенных исследований.

(C) Siberian Federal University. All rights reserved

This work is licensed under a Creative Commons Attribution-NonCommercial 4.0 International License (CC BY-NC 4.0).

* Corresponding author E-mail address: artemgolub91@gmail.com 
Ключевые слова: симплексный поиск, симплексный инвариантный метод, прямые методы оптимизации, дрейф целевой функции, оптимизация, адаптация, параметрическая адаптация.

Цитирование: Голуб, А.А. Оценка этапа поиска симплексного инвариантного метода / А.А. Голуб // Журн. Сиб. федер. ун-та. Техника и технологии, 2020. 13(2). С. 175-186. DOI: 10.17516/1999-494X-0214

\section{Введение}

Часто при решении задачи поиска и отслеживания дрейфующей цели приходится сталкиваться с рядом проблем, обусловленных неполной априорной информацией об объекте. Для решения подобных задач необходимо использовать такие методы, которые обеспечивают самонастройку в процессе работы.

В работе [1] раскрыто применение последовательного симплексного метода для решения задачи оптимизации, получены статистические характеристики поиска, построены несколько стратегий и вариантов алгоритма поиска, разработана методика их практического применения в задачах оптимизации реальных объектов. Использование симплексного инвариантного метода (СИМ) и инвариантного комплекс-метода (ИКМ) для решения задач поиска и отслеживания нестационарного экстремума отмечена в исследованиях [2-4]. В этих работах подчеркнута эффективность прямых методов поиска при работе в условиях неполной априорной информации. Основные принципы построения инвариантных к контролируемым возмущениям алгоритмов заключаются в следующем.

1. На каждом шаге поиска $k$ с целью получения оценок градиента $a_{i}$ и оценок зависимости возмущение-выход $b_{j}$ производится аппроксимация целевой функции выражением вида

$$
\hat{Q}(\mathbf{x}(k), \mathbf{z}(k))=\sum_{i=1}^{n}\left(a_{i}(k) \cdot x_{i}(k)\right)+\sum_{i=1}^{m}\left(b_{i}(k) \cdot z_{i}(k)\right),
$$

здесь $\mathbf{x}$ - вектор управляемых переменных; $\mathbf{z}$ - линеаризованный вектор контролируемых возмущений; $k$ - дискретное время; $n$ - количество управляемых переменных; $m$ - количество контролируемых возмущений.

2. На каждом шаге происходит вычитание вкладов возмущений в целевую функцию:

$$
C_{j}(k)=Q_{j}(k)-\sum_{i=1}^{m}\left(b_{i}(k) \cdot z_{i}(k)\right), j=1, \ldots, n+1,
$$

где $C_{j}$ - скорректированное значение целевой функции в $j$-й вершине текущего симплекса; $Q_{j}-$ измеренные значения целевой функции в $j$-й вершине текущего симплекса.

3. Выбор направления движения симплекса в пространстве происходит по соотношению скорректированных значений $C_{j}$.

В работе [5] рассмотрен вариант структурной адаптации прямого метода поиска. Суть такой адаптации заключается в использовании разных модификаций, в частности комплексметода на определенном этапе поиска.

В продолжение разработки адаптивных алгоритмов поиска необходимо рассмотреть подходы, обеспечивающие параметрическую адаптацию поисковых методов.

Для СИМ основным управляемым параметром может являться размер его ребра. Неверно выбранный размер ребра может привести к ряду неблагоприятных последствий. На рис. 1 изображены траектории движения экстремума и симплекса в процессе поиска и отслеживания.

$$
-176-
$$




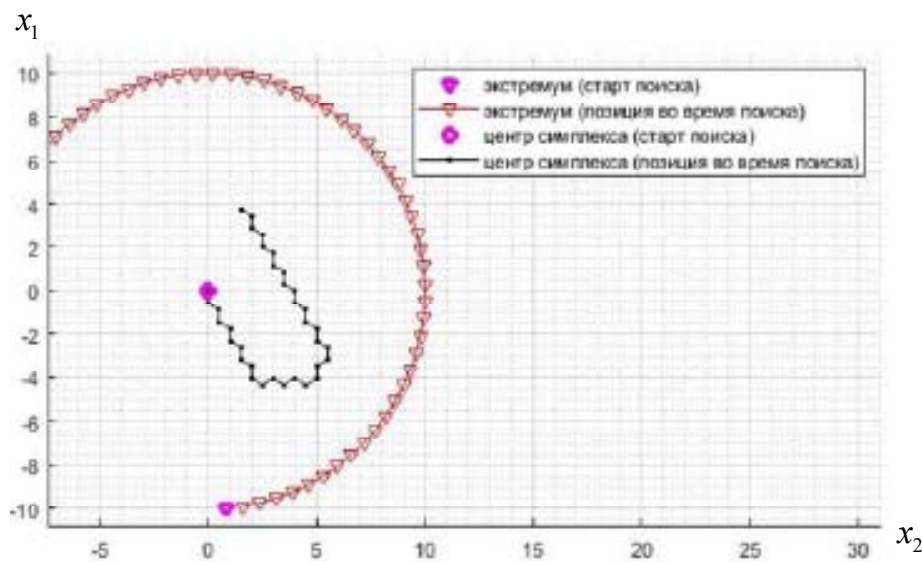

Рис. 1. Отслеживание экстремума (малый размер ребра симплекса)

Fig. 1. Tracking the extremum (small size of the edge of the simplex)

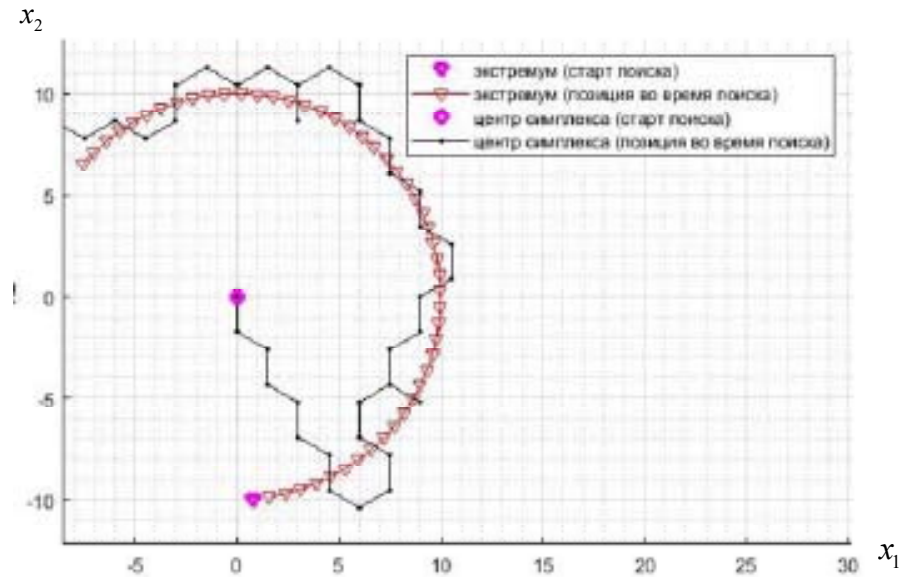

Рис. 2. Отслеживание экстремума (большой размер ребра симплекса)

Fig. 2. Tracking the extremum (big size of the edge of the simplex)

Если размер ребра будет задан небольшим, равным или меньше расстояния, на которое экстремум смещается за одну единицу дискретного времени, то, учитывая исходное отставание, симплекс не сможет настичь цель. На рис. 1 видно, что симплекс выдерживает общее направление движения, но из-за малого размера шага ему не удается догнать цель.

Рассмотрим обратную ситуацию: с заведомо большим шагом поиска (рис. 2).

На рис. 2 видно, что симплекс достиг дрейфующего экстремума, повторяет его траекторию движения. Но качество повторения траектории неидеальное: имеют место случаи повторных отражений и закручиваний симплекса вокруг одной из вершин. Это говорит о том, что скорость движения экстремума много меньше скорости движения симплекса, последний вынужден простаивать в околоэкстремальной области, пока экстремум сам не выйдет из текущей зоны локализации симплекса. 
Можно предположить, что существует такой размер ребра симплекса, при котором он будет отслеживать цель с минимальным отставанием. Также стоит отметить, что на первом этапе поиска (когда симплекс догоняет цель) размер ребра должен иметь возможность увеличиваться до максимально допустимого значения, чтобы как можно быстрее достичь экстремальной области. Для этого необходимо, чтобы размер ребра симплекса самостоятельно изменялся в процессе поиска и отслеживания. Законы, по которым могла бы происходить такая самонастройка, неочевидны ввиду малой априорной информации о самом объекте.

Цель данной работы - анализ характеристик, получаемых в процессе поиска и отслеживания экстремума на предмет выявления закономерностей, которые впоследствии можно будет использовать для оценивания этапа работы инвариантного симплекс-метода.

\section{Постановка задачи}

Рассмотрим задачу поиска и отслеживания экстремума нестационарной модели:

$$
Q(\mathbf{x}(k), \mathbf{z}(k)) \rightarrow \min _{x \in X}
$$

где $X=\left\{\mathbf{x}: \mathbf{x} \in E^{n}, x^{-} \leq x \leq x^{+}\right\}, x^{-}, x^{+}$- позиционные ограничения задачи; $E^{n}-n$-мерное евклидово пространство; $\mathbf{x}$ - вектор управляемых переменных; $\mathbf{z}(k)$ - вектор контролируемых возмущений; $k$ - дискретное время.

В качестве целевой функции примем модель следующего вида:

$$
Q(\mathbf{x}(k), z(k))=z(k)+\left(x_{1}(k)-R \sin (\omega \cdot z(k))\right)^{2}+\left(x_{2}(k)-R \cos (\omega \cdot z(k))\right)^{2},
$$

где $z(k)=v_{q} \cdot k, v_{q}-$ скорость вертикального дрейфа экстремума; $R$ - радиус траектории экстремума (горизонтальной составляющей); $\omega$ - угловая скорость дрейфа экстремума.

Такой выбор модели обусловлен тем, что в траектории движения экстремума будут как горизонтальная, так и вертикальная составляющие. Причем направление горизонтального дрейфа будет в течение процесса поиска постоянно меняться.

Зададим параметры модели таким образом, чтобы экстремум за одну единицу дискретного времени смещался в горизонтальной плоскости пространства поиска на расстояние, равное единице, другими словами, скорость дрейфа экстремума в горизонтальной плоскости будет равна $v_{\mathbf{x}}=1$. Это позволит представлять информацию о размере ребра симплекса и его скорости движения сразу в отношении к фактической скорости дрейфа цели.

Угловую скорость горизонтальной составляющей дрейфа можно выразить через радиус окружности, описываемой траекторией, и скоростью горизонтальной составляющей дрейфа:

$$
\omega=\arccos \left(1-\frac{v_{\mathbf{x}}^{2}}{2 R^{2}}\right) .
$$

Таким образом, при условии, что $v_{\mathbf{x}}=1$, параметры модели примем следующими: $v_{q}=1$; $R=50 ; \omega=0.02$.

Условимся, что поиск стартует вдали от экстремума. Так как горизонтальная составляющая траектории движения экстремума представляет собой окружность, то будет логичным, чтобы симплекс стартовал из равноудаленной от траектории дрейфа точки - центра этой окружности $\left(x_{1}=0, x_{2}=0\right)$. Количество шагов поиска ограничим значением $K=300$.

$$
-178-
$$




\section{Исследование характеристик}

Проведем несколько экспериментов с разным размером ребра симплекса. Рассмотрим несколько характеристик, полученных во время поиска:

- значения целевой функции на каждом шаге $Q(k)$;

- максимальное количество закручиваний вокруг одной из вершин симплекса на каждом шаге $M(k)$.

Дополнительно построим график отставания центра тяжести симплекса от цели на каждом шаге $E(k)$.

Для первого эксперимента примем ребро симплекса $L=1$ (рис. 3). В таком случае скорости движения симплекса вдоль координат $x_{1}$ и $x_{2}$ будут, соответственно, следующими:

$$
\begin{aligned}
& r_{x_{1}}=\frac{L}{2}=0.5, \\
& r_{x_{2}}=\frac{L \sqrt{3}}{3} \approx 0.58,
\end{aligned}
$$

что меньше скорости движения оптимума в горизонтальной плоскости $v_{\mathbf{x}}=1$.

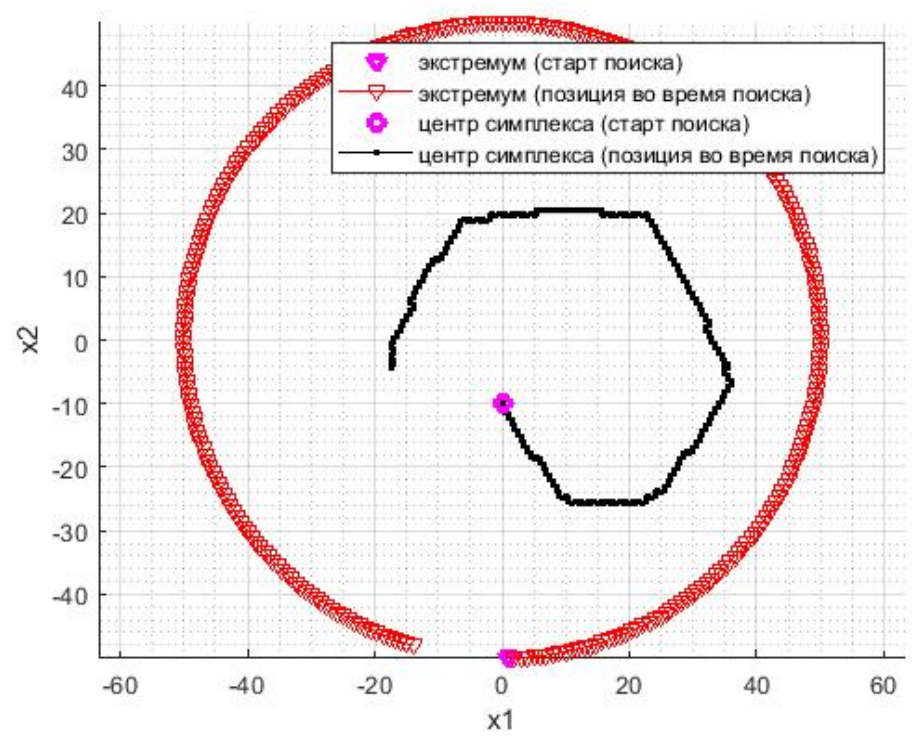

Рис. 3. Траектории движения экстремума и симплекса (малый размер ребра симплекса)

Fig. 3. Trajectories of extremum and simplex movements (small size of the edge of the simplex)

На рис. 4 видно, что зависимость $Q$ (значения целевой функции) повторяет характер зависимости отставания симплекса от цели $E$. Значения $Q$ уменьшались одновременно с сокращением расстояния до экстремума и росли с его увеличением. Для симплекса критерием закручивания вокруг одной из вершин [1] является превышение этим параметром значения

$$
M>1.65 n+0.05 n^{2}
$$


или для текущего случая при $n=2$

$$
M>3.5 \text {. }
$$

При проведении предыдущего эксперимента было зарегистрировано несколько случаев чрезмерного закручивания симплекса, но в целом количество последовательных вращений вокруг одной и той же вершины симплекса не превышало значения 3. Это говорит о том, что симплекс не локализовался в процессе поиска - все время происходило его движение.

На рис. 5, 6 показаны результаты эксперимента с размером ребра $L=4$, дающим скорость перемещения симплекса выше, чем скорость горизонтального дрейфа $v_{\mathbf{x}}=0.8: r_{x_{1}}=2, r_{x_{2}} \approx 2.3$.

На рис. 6 заметно влияние чрезмерного закручивания на расстояние до экстремума - в то время, когда симплекс вращается на месте, экстремум успевает удалиться. Также в некоторой степени влияние закручивания распространяется на характеристику $Q$ - на графике в те же моменты времени наблюдаются небольшие скачки.

В данном эксперименте симплекс достигает своей цели примерно к 25-му шагу, что можно увидеть на графике отставания. Характеристика $Q$ на этом этапе также имеет схожий характер изменения. После того как симплекс достиг экстремальной области, во время следования за оптимумом периодически происходит чрезмерное закручивание симплекса. Можно предположить, что это связано с тем, что размер шага симплекса много больше шага экстремума и первый вынужден простаивать в некоторой локации до тех пор, пока оптимум не выйдет за ее пределы.

Если увеличить количество шагов поиска до $K=1000$, характеристики будут иметь следующий вид (рис. 7).

После достижения околоэкстремальной области симплекс поддерживает некоторый минимальный разрыв с целью, значения $Q$ плавно растут - это свидетельство наличия вер-
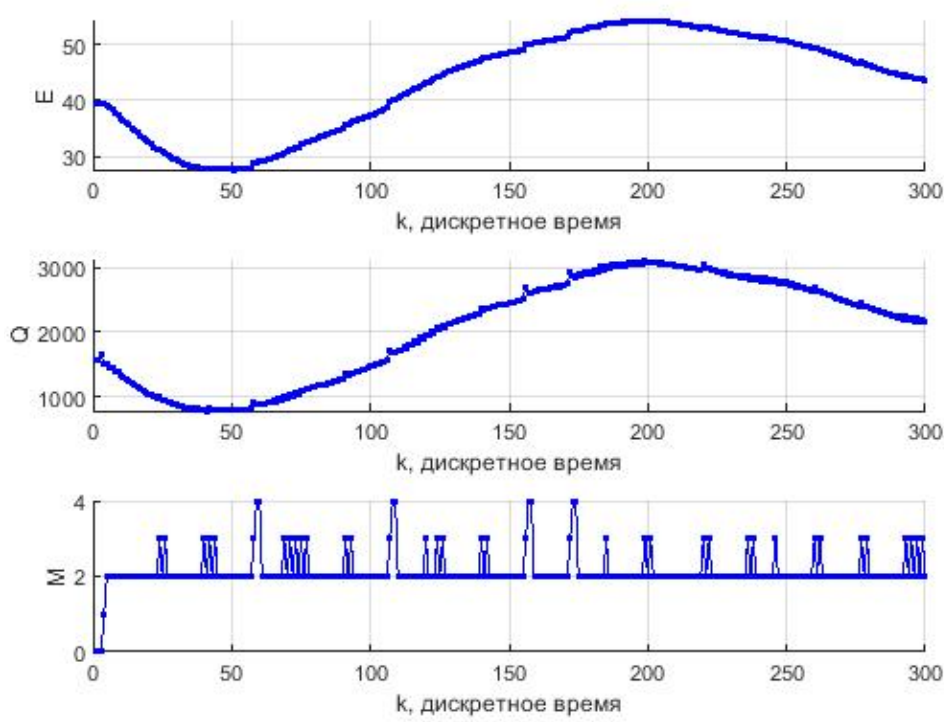

Рис. 4. Характеристики процесса поиска (малый размер ребра симплекса)

Fig. 4. Characteristics of the search process (small size of the edge of the simplex)

$$
-180-
$$




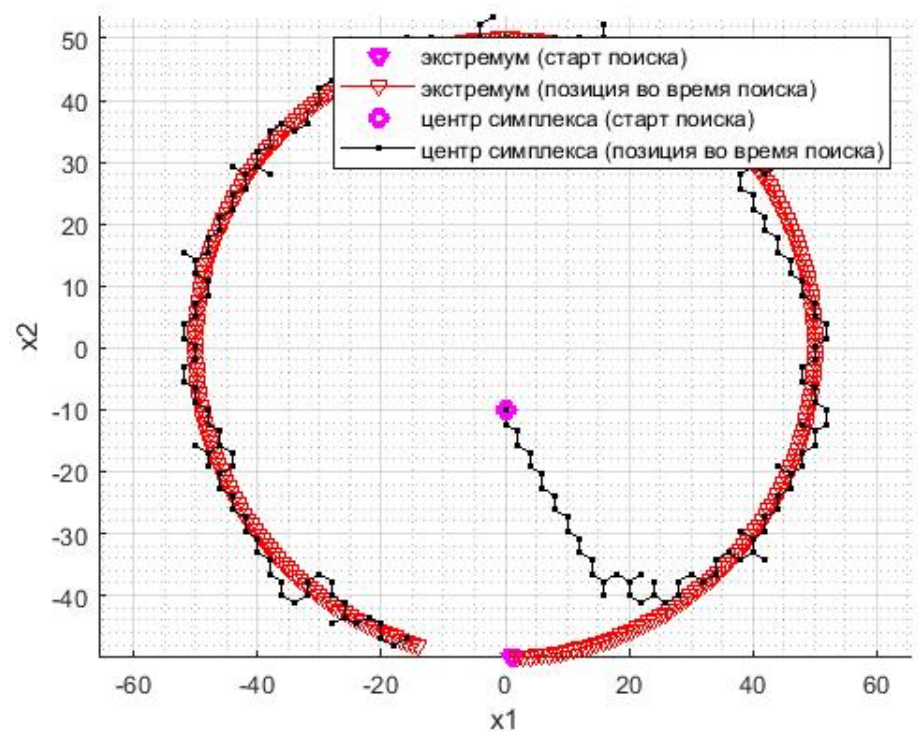

Рис. 5. Траектории движения экстремума и симплекса (большой размер ребра симплекса)

Fig. 5. Trajectories of extremum and simplex movement (big size of the edge of the simplex)
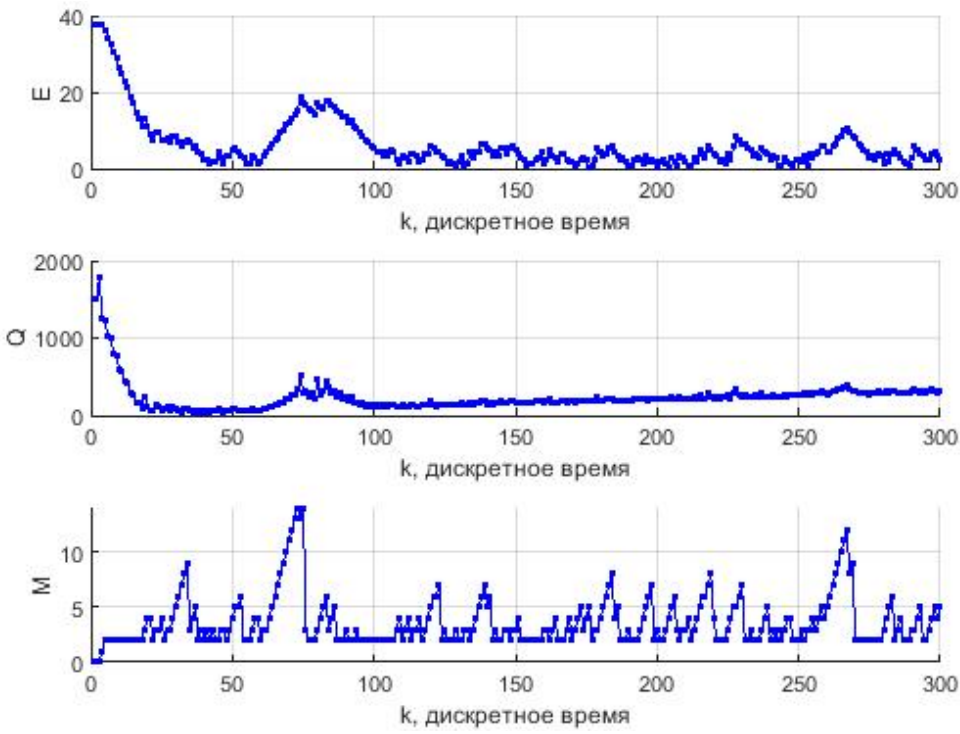

Рис. 6. Характеристики процесса поиска (большой размер ребра симплекса)

Fig. 6. Characteristics of the search process (big size of the edge of the simplex)

тикального дрейфа целевой функции. Время от времени происходит закручивание симплекса.

Полученной информации достаточно, чтобы сделать вывод о том, что на разных этапах поиска и при разных размерах ребра симплекса характеристика $Q$ имеет отличительные признаки. Следовательно, можно допустить, что после анализа этих признаков удастся синтезировать правила, которые позволили бы оценивать этапы поиска: этап спуска, когда симплекс догоняет 

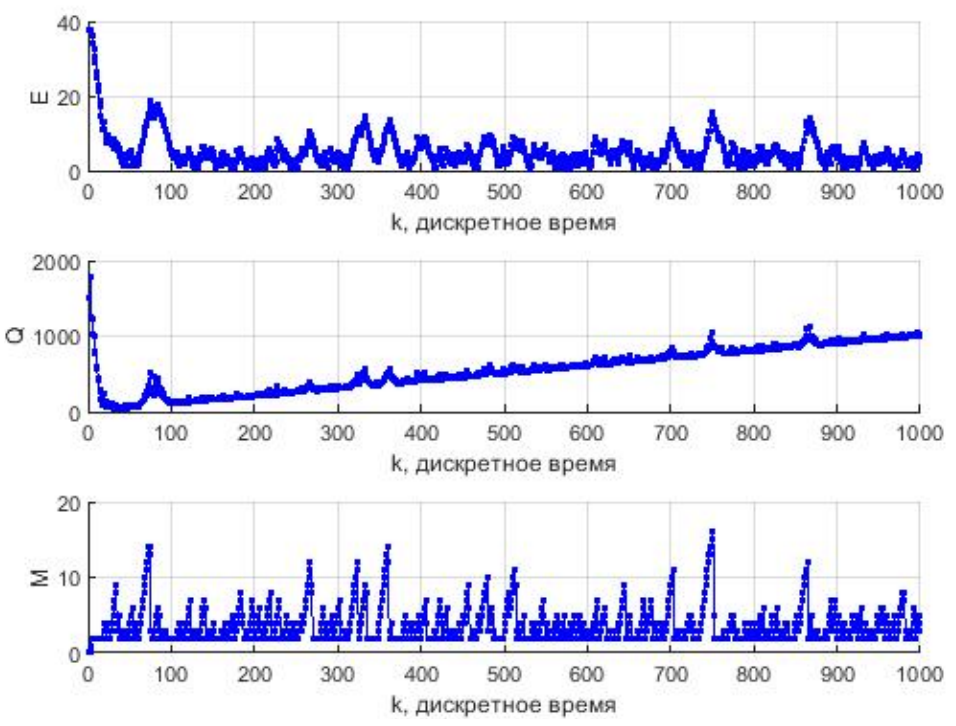

Рис. 7. Характеристики процесса поиска (большой размер ребра симплекса, 1000 шагов)

Fig. 7. Characteristics of the search process (big size of the edge of the simplex, 1000 steps)

экстремум, и этап отслеживания, когда симплекс двигается в околоэкстремальной области. Для каждого из этапов необходима своя стратегия управления размером симплекса.

На этапе спуска необходимо увеличивать размер симплекса до максимально допустимого с иелью наискорейшего достижения зоны оптимума.

Во время отслеживания необходимо уменьшать размер ребра до того уровня, чтобы скорости движения симплекса и экстремума уравнялись. Допускается менять размер ребра во время отслеживания для того, чтобы скомпенсировать отставание, вызванное помехами или некорректной оценкой скорости дрейфа.

Рассмотрим подробнее особенности поведения $Q$. Имеет смысл подвергнуть анализу не только исходную характеристику, но и некоторые производные, которые можно получить на ее основе.

Для удобства анализа и возможного последующего применения эти характеристики следует представить в сглаженном виде, например, использовав скользящее среднее. Условимся, что для расчета скользящего среднего будет применяться окно глубиной, равной $N$ шагам поиска. На этапе поиска до шага $N$ будет использоваться окно с глубиной, равной текущему шагу. Скользящее среднее на каждом шаге $k$ для $Q$ рассчитывают следующим образом:

$$
\bar{Q}(k)=\frac{1}{N} \cdot \sum_{i=k-N+1}^{k} Q(i) .
$$

В качестве одной из производных характеристик можно рассмотреть разности первого и второго порядков полученного ряда значений $\bar{Q}(k)$ :

$$
\begin{aligned}
\Delta \bar{Q}(k) & =\bar{Q}(k)-\bar{Q}(k-1), \\
\Delta^{2} \bar{Q}(k)=\Delta \bar{Q}(k)-\Delta \bar{Q}(k-1) . & -182-
\end{aligned}
$$



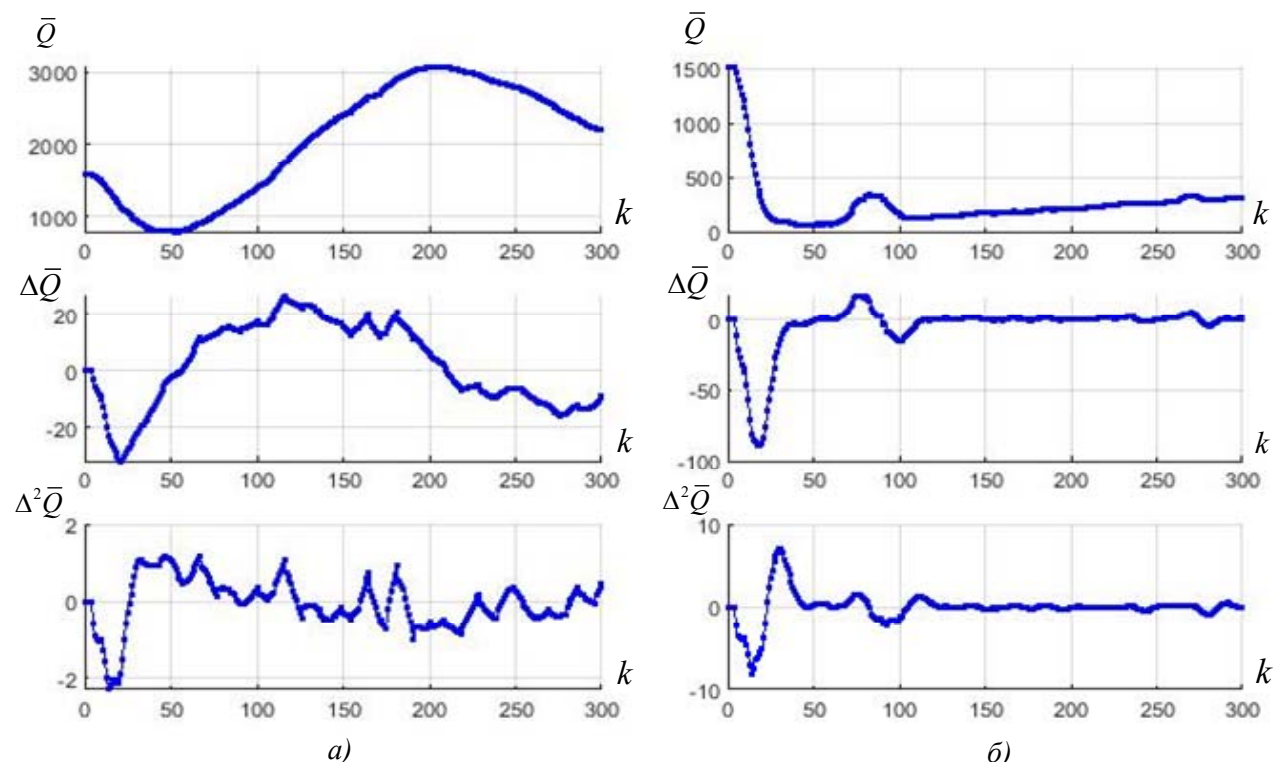

a)

б)

Рис. 8. Характеристики процесса поиска с разным ребром симплекса: $\mathrm{a}-L=1 ; \sigma-L=4$

Fig. 8. Characteristics of the search process with a different edge of the simplex: $a-L=1 ; \sigma-L=4$

Характеристики $\bar{Q}(k), \Delta \bar{Q}(k)$ и $\Delta^{2} \bar{Q}(k)$ изображены на рис. 8. Параметры модели и время моделирования прежние. Эксперименты проведены с малым и большим размерами ребра симплекса.

На рис. $8 a$ изображены характеристики поиска с большим ребром симплекса. На разных этапах поиска характер зависимостей $\Delta \bar{Q}$ и $\Delta^{2} \bar{Q}$ отличается.

На этапе спуска происходит падение значений $\bar{Q}$ - симплекс движется по направлению к экстремуму. А так как $\Delta \bar{Q}$ и $\Delta^{2} \bar{Q}$ можно считать соответственно скоростью и ускорением изменения характеристики $\bar{Q}$, то их вид будет это отражать.

На этапе отслеживания $\Delta \bar{Q}$ и $\Delta^{2} \bar{Q}$ перестают резко изменяться. Допускаем, что на этом этапе симплекс следует за экстремумом с близкой последнему скоростью, значит, значения $\Delta \bar{Q}$ можно расценивать как оценку скорости вертикального дрейфа. А так как в данном эксперименте скорость вертикального дрейфа постоянна, то таким же будет и характер $\Delta \bar{Q}$.

Зависимость $\Delta^{2} \bar{Q}$ на этапе отслеживания принимает близкие к нулевым значения ввиду того, что колебания $\Delta \bar{Q}$ минимальны.

Примерно на 75-м шаге поиска наблюдается резкий всплеск на всех характеристиках - это следствие локализации симплекса в некоторой области.

На рис. $8 б$ изображены характеристики поиска с малым ребром симплекса. В отличие от предыдущего эксперимента здесь не наблюдается признаков этапа отслеживания: нет постоянства характеристик $\Delta \bar{Q}$ и $\Delta^{2} \bar{Q}$. При малом шаге симплекс не может достичь экстремума (см. рис. 3).

Дополнительно можно рассмотреть другие характеристики поиска: разность усредненного значения $\bar{Q}$ на шаге $k$ и минимального $\bar{Q}$ за всю предысторию поиска

$$
-183-
$$




$$
\Delta_{\min } \bar{Q}(k)=\bar{Q}(k)-\min (\bar{Q})
$$

а также конечную разность, полученную на основе (13)

$$
\Delta_{\min }^{2} \bar{Q}(k)=\Delta_{\min } \bar{Q}(k)-\Delta_{\min } \bar{Q}(k-1) .
$$

Характеристики $\bar{Q}(k), \Delta_{\min } \bar{Q}(k)$ и $\Delta_{\min }^{2} \bar{Q}(k)$ изображены на рис. 9. Параметры модели и время моделирования прежние. Эксперименты проведены с малым и большим размерами ребра симплекса.

На рис. $9 a$ изображены характеристики поиска с большим ребром симплекса. На этапе спуска, в отличие от $\Delta \bar{Q}$ и $\Delta^{2} \bar{Q}$, полученных в предыдущем эксперименте (рис. $8 a$ ), зависимости $\Delta_{\min } \bar{Q}$ и $\Delta_{\min }^{2} \bar{Q}$ принимают нулевые значения. На этапе отслеживания $\Delta_{\min } \bar{Q}(k)$ начинает расти с некоторой постоянной скоростью - связь с постоянством скорости вертикального дрейфа. Характеристика $\Delta_{\min }^{2} \bar{Q}(k)$ на этом этапе начинает принимать некоторое, близкое к постоянному, значение.

На 75-м шаге поиска также наблюдается резкий всплеск - следствие закручивания симплекса.

На этапе спуска $\Delta_{\min } \bar{Q}(k)$ и $\Delta_{\min }^{2} \bar{Q}(k)$ принимают нулевые значения. Это объясняется тем, что $\Delta_{\min } \bar{Q}(k)$ рассчитывается относительно минимального значения $\bar{Q}$ за всю предысторию поиска, и, если учитывать, что симплекс с каждым шагом продвигается в направлении экстремума, любое новое значение $\bar{Q}$ будет минимальным.

На рис. 96 изображены характеристики поиска с малым ребром симплекса. В отличие от предыдущего эксперимента по виду характеристик нельзя увидеть признаков этапа отслеживания: $\Delta_{\min } \bar{Q}(k)$ и $\Delta_{\min }^{2} \bar{Q}(k)$ не имеют постоянного характера изменения.

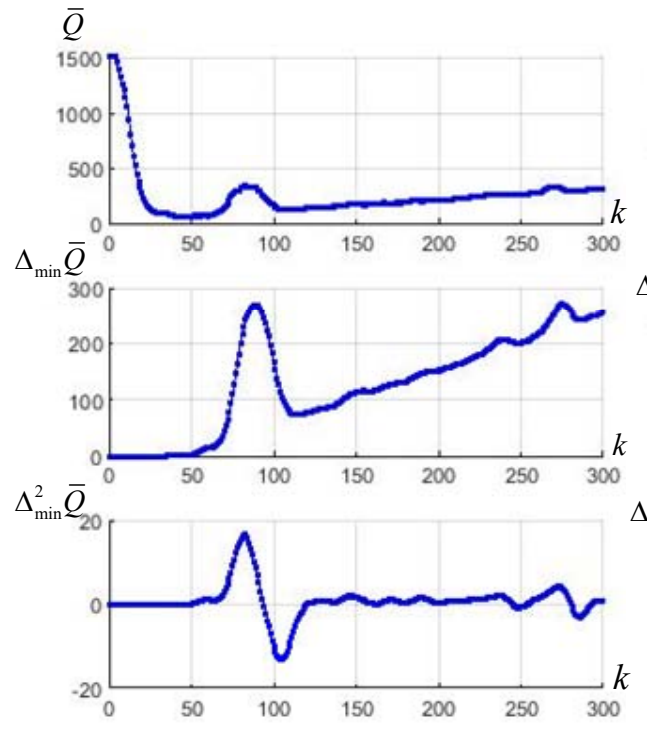

a)
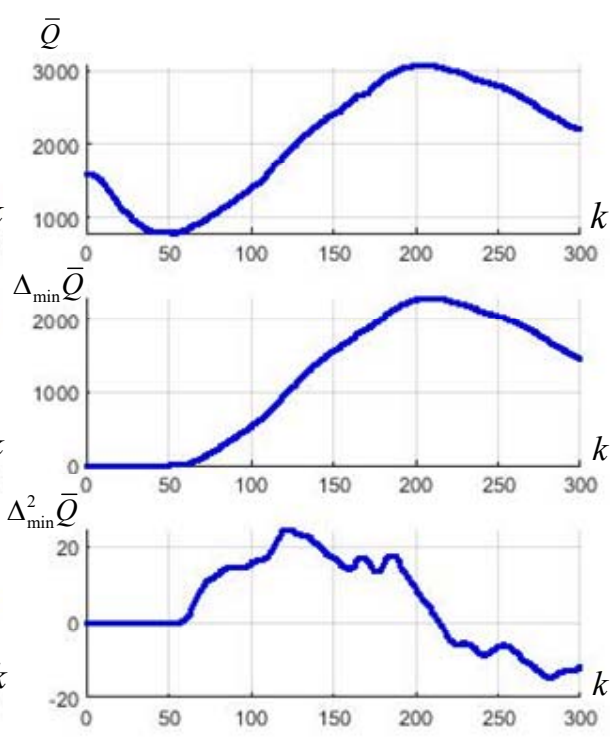

б)

Рис. 9. Характеристики процесса поиска с разным ребром симплекса: а $-L=4 ; 6-L=1$

Fig. 9. Characteristics of the search process with a different edge of the simplex: a $-L=4 ; \sigma-L=1$ 
Таблица 1. Признаки этапов поиска

Table 1. Signs of the search stages

\begin{tabular}{|c|c|c|c|c|}
\hline \multirow{2}{*}{ Характеристика } & \multicolumn{2}{|c|}{ Малое ребро симплекса } & \multicolumn{2}{c|}{ Большое ребро симплекса } \\
\cline { 1 - 5 } & Спуск & Отслеживание & Спуск & Отслеживание \\
\hline$\Delta \bar{Q}$ & $<0, \neq$ const & Нет данных & $<0, \neq$ const & $\geq 0,=$ const \\
\hline$\Delta^{2} \bar{Q}$ & $\neq$ const & Нет данных & $\neq$ const & $\approx 0,=$ const \\
\hline$\Delta_{\min } \bar{Q}$ & $\approx 0,=$ const & Нет данных & $\approx 0,=$ const & $\geq 0$ \\
\hline$\Delta_{\min }^{2} \bar{Q}$ & $\approx 0,=$ const & Нет данных & $\approx 0,=$ const & $\neq$ const \\
\hline
\end{tabular}

Таблица 2. Правила оценки этапа поиска

Table 2. Estimation rules of the search stages

\begin{tabular}{|c|c|}
\hline Условия & Этап работы \\
\hline$\Delta \bar{Q} \neq$ const \& $\Delta_{\min } \bar{Q}=$ const \& $\Delta_{\min } \bar{Q} \approx 0$ & Спуск \\
\hline$\Delta \bar{Q}=$ const $\& \Delta_{\min } \bar{Q} \geq 0 \& \Delta_{\min }^{2} \bar{Q} \neq$ const & Отслеживание \\
\hline
\end{tabular}

Так как симплекс с малым ребром не имеет возможности достичь области дрейфующего экстремума, то в этом случае этапа отслеживания цели не будет как такового. На рис. $4,8 a$ можно заметить, что спуск чередуется с отставанием от цели.

\section{Оценивающие правила}

Полученную в ходе анализа информацию сведем в таблицу признаков (табл. 1).

На основе части соответствий признаков определенных этапов поиска в табл. 2 синтезируем правила, которые позволят произвести оценку этапа поиска.

Получая информацию о текущем в этапе, можно выбирать определенную стратегию поиска для достижения максимальной эффективности работы.

\section{Заключение}

В данной статье рассмотрен метод оценки этапа работы симплексного инвариантного метода.

Проведен анализ характеристик процесса поиска и отслеживания экстремума нестационарной статической модели. На основе выявленных закономерностей построены правила, при помощи которых можно определить текущий этап: поиск экстремума или его отслеживание.

Информацию о текущем этапе поиска можно применять для выбора оптимальной стратегии; например, на этапе спуска желательно использовать максимально допустимый размер ребра симплекса для быстрого достижения экстремальной области, а во время отслеживания необходимо регулировать размер ребра так, чтобы скорости движения симплекса и экстремума уравнялись. 


\section{Список литературы / References}

[1] Дамбраускас А.П. Симплексный поиск. М.: Энергия, 1979. 176 с. [Dambrauskas A.P. Simplex search. Moscow, Energia, 1977 (in Russian)]

[2] Масальский Г.Б. Разработка и исследование инвариантных методов поиска в задачах оптимизации технологических процессов. М., 1977. 224 с. [Masalsky G.B. Development and research of invariant search methods in problems of optimization of technological processes. Moscow, 1977 (in Russian)]

[3] Голуб А.А. Решение задачи поиска и отслеживания дрейфующего экстремума инвариантным комплекс-методом. Робототехника и искусственный интеллект. Материалы IX Всероссийской научно-технической конференции с международным участием (2. Железногорск, 2 декабря 2017 г.) Науч. ред. В.А. Углева. Красноярск: ЛИТЕРА-принт, 2017. С. 97-102 [Golub A.A. Solving the problem of searching and tracking drift extremum using the invariant complex method. Robotics and artificial intelligence. Materials of the IX all-Russian scientific and technical conference with international participation (Zheleznogorsk, December 2, 2017). Krasnoyarsk, Litera-print, 2017, 97-102 (in Russian)]

[4] Голуб А.А. Модернизация комплекс-метода для задач робототехники. Молодежь и наука. Сборник материалов IX Всероссийской научно-технической конферениии студентов, аспирантов и молодых ученых с международным участием. Красноярск: Сиб. федер. ун-т., 2013 [Golub A.A. Modernization of the complex-method for the tasks of robotics. Youth and science. A collection of materials of the IX all-Russian scientific and technical conference of students, graduate students and young scientists with international participation. Krasnoyarsk, Siberian Federal University, 2013 (in Russian)]

[5] Голуб А.А. Структурная адаптация прямого метода поиска. Проспект Свободный-2018. Материаль Международной студенческой конферениии (Красноярск, 23-27 апреля 2018 г.). Красноярск: Сиб. федер. ун-т., 2018. С. 825-828 [Golub A.A. Structural adaptation of the direct search method. Prospekt Svobodny - 2018. Materials of the International Student Conference (Krasnoyarsk, April 23-27, 2018). Krasnoyarsk, Siberian Federal University, 2018, 825-828 (in Russian)] 